\title{
Corporate Investment Dynamic Control System Based on Chaos Cycle Perturbations
}

\author{
Yanyan Gao ${ }^{*}, 1$ and Guoliang Huang ${ }^{2}$ \\ School of Management, China University of Mining and Technology Xuzhou, 221000, Jiangsu, China.
}

Received 22 September 2014; Revised 4 October 2014; Accepted 5 November 2014

\begin{abstract}
It exists some issues such as the low predict accuracy and a bad convergence performance to predict business investment with BP neural network algorithm. This paper presents a predictive model of business investment based on improved artificial bee colony and chaos periodic disturbance optimizing BP neural network algorithm. At first, use Boltzmann selection strategy and group behaviour control strategy to optimize the artificial bee colony algorithm, and then use the improved algorithm to transform BP neural network algorithm's optimized parameters into optimization process of artificial bee colony algorithm to reduce the training error of the original algorithm. Finally, use chaotic optimized Logistic mapping enables BP neural network out of the local minimum point in the training process based on secondary chaotic cycle perturbation strategies. Simulation results show that the proposed predictive model of investment in the enterprise based on improved artificial bee colony and chaos periodic disturbance optimizing BP neural network algorithm shows higher predict accuracy and better convergence than normal BP neural network algorithm.
\end{abstract}

Keywords: Dynamic business investment control, investment prediction model, chaos periodic disturbance, improved artificial bee colony algorithm, optimizing BP neural network.

\section{Introduction}

With the deepening of reform and opening up, the reformation of economic, the gradual improvement of the market economy, China's economy is growing at an unprecedented rate to achieve a sustained and rapid growth [1]. The development and deepening of economic reform has brought the full range of economic investment in China's state capital, collective capital and individual capital [2]. The establishment of corporate internal control mechanisms can regulate the investment market better, protect investors' investment income effectively, and constraint operators' manufacturing operate management behavior [3]. However, for big companies which hold a large number of idle funds or operating venture capital, looking for a good investment and circulating income funds to maximize profits is particularly important [4]. So the risk control for business investment is significant.

Experts and scholars from home and abroad have been studied many theories on business investment control indepth and extensively. Economic chaos is the basic research, and only on this basis we can clear economic chaos characteristics to establish the correct chaotic economic model [5]. Bo Er'ding proved that optimal economic growth

* E-mail address: cumt_gjf@163.com ISSN: 1791-2377 @ 2015 Kavala Institute of Technology. All rights reserved. track would produce chaos under certain conditions, and further showed the irregular fluctuations in economic phenomena are determined by inner economic system which is subject to the results of joint action within market forces, technological change and the propensity to consume [6]. From the acceleration econometric model of describing the two-stage of the capitalist market economy and the long wave investment model of socialist planned economy, Lu Sai indicated a chaotic may appear in centrally planned socialist economy [7]. Huang Xiaoyuan studied the conditions of dynamic economic growth equation produce chaotic under unilateral and bilateral restricted [8]. Xie Li proved that the existence of chaotic behavior in economic dynamic system based on the analysis of economic growth optimal control instance [9]. Yu Fawen discussed the application of chaos theory in nonlinear economic system, focusing on the statistical tests of economic structure and the control of economic structure [10]. Quan Lin applied chaos theory into economic cycle theory, discussed chaos theory related to the economic cycle model in detail and gave the application examples which solved the problems that the traditional methods of dynamic systems theory can not explain [11]. Block found the low-dimensional chaotic attractors from high frequency data produced by equity securities, foreign exchange, futures markets and studied existence of the economic chaos from empirical data [12]. Chen ping found the empirical evidence of economic chaos from the American currency index, established soft elastic 
oscillator model describing complex economic fluctuations, also found the effective method of decomposing the economic growth and volatility, and decomposing noise and signal. Thereby he found widespread evidence of economic chaos from a variety of representative of U.S. macroeconomic indicators [13]. Wang Chunfeng applied chaos economic theory into the analysis of macroeconomic situation, confirmed China's macroeconomic system appear chaotic, and pointed out that the reason for the occurrence of deflation was the imperfect chaotic economic system internal mechanism which reduced the stability and antijamming capability of the system [14]. Fan Ruguo studied deeply the nonlinear mechanism of financial crisis from chaos theory point. The Asian financial crisis is considered a financial chaotic behaviour [15]. Dai Guoqiang used chaos theory to discuss nonlinear problem in the economic system and to explore the policy implications of their implicit [16]. Ding Xiaofeng applied chaos economic theory to chaos economic model of transform company to intensive targets and the accumulation of knowledge during economic growth process and others [17].

In this paper, for business investment demand, we proposed BP neural network algorithm based on improved artificial bee colony and chaos periodic disturbance optimizing to improve the predict accuracy of using BP neural network algorithm to predict business investment.

\section{The Business Investment Prediction Model based on Neural Network Enterprise}

Since the BP neural network's highly nonlinear fitting capability and self-organizing ability, we use it to model and forecast business investment control system.

The business investment prediction model based on neural network enterprise is composed by the input layer, hidden layer and output layer. The input layer is previous years' data of business investment. The output layer is the predicted data of business investment. Assume $N$ sample set $\left\{(x(t), y(t)) \mid x \in R^{m}, y \in R^{n}, t=1,2, \ldots, N\right\}$ to be discrete set of time series, and then use BP neural network to map its highly non-linear from input to output: $F: R^{m} \rightarrow R^{n}$. Put the sample of all business investment into training sample $\phi_{1}$ and test samples $\phi_{2}$ :

$$
\begin{aligned}
& \phi_{1}=\left\{(x(t), y(t)) \mid x \in R^{m}, y \in R^{n}, t=1,2, \ldots, N_{1}, N_{1} \leq N\right\} \\
& \phi_{2}=\left\{(x(t), y(t)) \mid x \in R^{m}, y \in R^{n}, t=N_{1}+1, N_{2}+2, \ldots, N\right\}
\end{aligned}
$$

Firstly, using corporate investment data training sample $\phi_{1}$ to establish mapping relationship, then see the BP neural network for business investment data test samples $\phi_{2}$ whether can give the correct inputs and outputs or not, and if it can, we say that the model can be used for business investment forecast in practice. For such a time series mapping is established, it can be used as an input node of $m$, the output node of $n$, hidden nodes of $p$ for a threelayer BP neural network to achieve. The relationship between the input and output of the network is as follows:

$$
\hat{y}_{k}(t)=\sum_{j=1}^{p} v_{j k} \cdot f\left[\sum_{i=1}^{m} w_{i j} \cdot x_{i}(t)+\theta_{j}\right]+r_{k}
$$

$f(x)=\frac{1}{1+e^{-x}}, k=1,2, \ldots, n, t=1,2, \ldots, N_{1}, x_{i}$ is the input layer, $\hat{y}_{k}$ is the output layer, $w_{i j}$ is the weight from the input layer nodes $j$ to the output layer nodes $k, \theta_{j}$ is the threshold at $j, r_{k}$ is the threshold at $k, f$ is the activation function.

Assume the total error in BP neural network is less than $\varepsilon_{1}$, there is:

$$
E_{1}=\frac{1}{2} \sum_{t=1}^{N_{1}} \sum_{k=1}^{n}\left[y_{k}(t)-\hat{y}_{k}(t)\right]^{2} \leq \varepsilon_{1}
$$
is:

Assume test sample error is less than the average, there

$$
E_{2}=\frac{1}{N-N_{1}} \sum_{t=N_{1}}^{N} \sum_{k=1}^{n}\left[y_{k}(t)-\hat{y}_{k}(t)\right]^{2} \leq \varepsilon_{2}
$$

The learning process of the business investment prediction model based on BP neural network enterprise is formed by the forward calculation process and the error back propagation process. Learning steps are as follows:

1. If the initialization input learning rate $\alpha, \beta$, given the maximum learning error (convergence precision) $\varepsilon$; give input layer to the hidden layer connection weights $\omega_{i n}$, hidden layer to output layer connection weights $T_{q i}$ and thresholds $\theta_{i}, \theta_{q}$ random value in $(-1,+1)$.

2. BP neural network provides a set of learning samples, in order to not affect business investment prediction model's learning performance by the absolute value of the input node, firstly normalized variables:

$Y=\frac{X-X_{\min }}{X_{\max }-X_{m m}}$

$\mathrm{Y}$ is the normalized value, $\mathrm{X}$ is true value, $X_{\max }, X_{\text {min }}$ the maximum and minimum values of variables. Quantify the input vectors and target vectors into $[-1,1]$.

3. Each mode performs $\left(A_{m}, C_{m}\right)(m=1,2, \ldots, k)$ the following actions:

Firstly, calculate the weighted input and output values of every layer and hidden layer weighted input:

$$
n e t_{t}=\sum_{n} w_{i n} \alpha_{n}-\theta_{i}
$$

The transfer function is the type of $(0,1) S$ function. As the following equation:

$$
f(x)=\frac{1}{1+e^{-x}}
$$


Then, weighted inputs the output layer:

$n e t_{q}=\sum_{q} T_{q i} y_{i}-\theta_{q}$

Calculate every layer's error, set the input layer node error as $\delta_{q}=-\left(t_{q}-C_{q}\right) f\left(\right.$ net $\left._{q}\right)$, so:

$\frac{\partial E}{\partial T_{q i}}=-\alpha \delta_{q} y_{i}$

Set the hidden layer node error as:

$\delta_{i}=f\left(\right.$ net $\left._{i}\right) \cdot \sum_{q} \delta_{q} T_{q i}$

So,

$\frac{\partial E}{\partial \omega_{i n}}=-\beta \delta_{i} \alpha_{n}$

Finally adjust the weights on each side, as amended weights $\Delta T_{q i}, \Delta \omega_{i n}$ proportional to the error function along the gradient descent,

$$
\begin{aligned}
& \Delta_{q i}=-\eta \delta_{q} y_{i} \\
& \delta_{q}=\left(t_{q}-C_{q}\right) \cdot f\left(\text { net }_{q}\right) \\
& \Delta \omega_{i n}=-\eta^{\prime} \frac{\partial E}{\partial \omega_{i n}}=\eta^{\prime} \delta_{i} a_{n} \\
& \delta_{i}=f^{\prime}\left(\text { net }_{i}\right) \sum_{q} \delta_{q} T_{q i}
\end{aligned}
$$

Use the above method to build business investment prediction model, to conduct a simulation example, and the results are as follows:

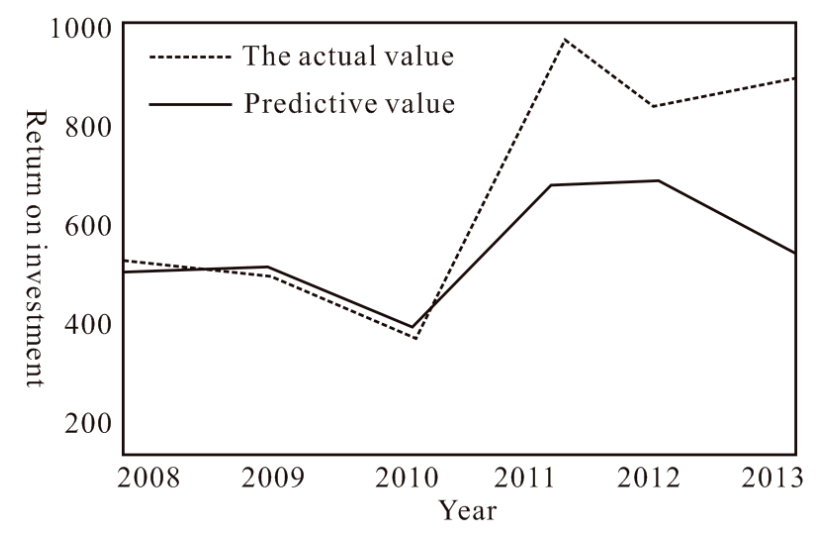

Fig. 1. Business investment forecast results.

Seen from the figure, although the fitting degree of BP neural network and training data is good, with the increase of the iteration, the prediction accuracy decreased significantly. Therefore, this paper use improved artificial bee colony and chaotic cycle perturbation to optimize it.

\section{Improved BP Neural Network based on Dual Optimization}

\subsection{Improvement of Artificial Bee Colony Algorithm}

For an optimization problem, artificial bee colony algorithm first generates a random initialization containing $m$ solutions of the initial population ( $m$ is also the number of hire bee or observe bee). Each solution represents with $D$ dimensional vector, $i$ is represented as $x_{i}=\left(x_{i 1}, x_{i 2}, \ldots, x_{i D}\right)$, $i=1,2, \ldots, m$. Then, hire bee produces a new nectar location around its nectar based on their memory of the nectar location.

$x_{i j}=x_{i j}+\Phi_{i j}\left(x_{i j}-x_{k j}\right)$

where $k$ is random selected from $\{1,2, \ldots, m\}, k \neq i$, $j \in\{1,2, \ldots, m\}$, and $\Phi_{i j}$ is random selected from $[-1,1]$.

The observe bee evaluate the nectar containing nectar information provided by hire bee and choosing nectar according to the following formula:

$p_{i}=\frac{f i t_{i}}{\sum_{j=1}^{m} f i t_{j}}$

where $f i t_{i}$ represents the amount of honey in the $i$ nectar.

In the algorithm, if a bee colony found the amount of honey is still at a low level and does not increase after "limit" times of iterative, the nectar will be abandoned. The abandoned nectar is:

$x_{i}^{j}=x_{\min }^{j}+\operatorname{rand}(0,1)\left(x_{\max }^{j}-x_{\min }^{j}\right)$

where $x_{\max }^{j}, x_{\min }^{j}$ are respectively the maximum and minimum $j$.

Applying artificial bee colony algorithm into artificial neural networks exist questions such as convergence is too earlier and falling into local optimal solution, then this paper optimizes it at first:

1. Dynamic selection of the target point.

In selecting the target point, the fire bee generate a new nectar around its nectar based on memory of the nectar information by following formula:

$v_{i j}=x_{i j}+\Phi_{i j}\left(x_{i j}-x_{k j}\right)$

Here, the fitness of nectar can be calculated by the average honey of individual and the distance between the individual bee and nectar:

$f\left(y_{i}\right)=\alpha \sqrt{y_{i 1}^{2}+y_{i 2}^{2}+\ldots+y_{i d}^{2}}+\beta \frac{S}{n}$

After complete the search, the fire bee tell the observation bee location and fitness of the nectar. The observation bees use Boltzmann selection strategy to choose nectar according to fitness following formula: 


$$
p_{i}=\frac{\exp \left(f_{i} / T\right)}{\sum_{i=1}^{S N} \exp \left(f_{i} / T\right)}, T=T_{0}\left(0.99^{0-1}\right)
$$

where $f_{i}$ is the solution of $i . p_{i}$ is the probability of nectar $x_{i}$.

2. The control of group behavior.

After choosing nectar based on Boltzmann selection strategy, the observation see choose $n$ individuals from the same nectar $y_{j j}$ form a small colony, planning path through information sharing mechanisms to avoid individual collisions.

After initializing each individual bee position $x_{i}$, velocity $v_{i}$, individual extreme $P_{b e s t}$ and global extremes $g_{\text {best }}$, calculate individual fitness $f\left(x_{i}\right)$, based on $f\left(x_{i}\right)=\alpha d+\beta f\left(y_{i j}\right)$, which is determined by the distance $d$ and the fitness $f\left(y_{i j}\right)$ of the individual and nectar $y_{i j}$. At the same time, the merits of target position and the distance between individual and target position are take into account. $\alpha, \beta$ are the impact factor. Then update the position and speed according to the following formula to select the optimal path:

$$
\begin{gathered}
v_{i d}=\omega v_{i d}+c_{1} \operatorname{rand}\left(P_{i d}-x_{i d}\right) \\
+c_{2} \operatorname{rand}\left(P_{g d}-x_{i d}\right)
\end{gathered}
$$

$$
x_{i d}=x_{i d}+v_{i d}
$$

where $\omega$ is the inertia weight, $c_{1}$ is acceleration weighting coefficient, $c_{2}$ is global acceleration weighting coefficient, usually $c_{1}=c_{2}=2$; rand is random number in $[0,1]$. In the calculation process, if $v_{i j}>v_{\max }$, then $v_{i j}=v_{\max }$.

\subsection{BP Neural Network Based on the Improved Artificial Bee Colony}

The optimization parameters of neural networks can be transformed into a food source in bee colony algorithm, so the food source can be expressed as:

$$
X=X\left(v_{11}, \ldots, v_{I 1}, \mu_{1}, \ldots, v_{1 J}, \mu_{J}, \ldots, w_{J K}, \theta_{k}\right)
$$

Among them, $v_{1 j}, v_{2 j}, \ldots, v_{I j}, \mu_{j}$ is the connection weights between the input node and the $j$-rd hidden layer nodes and the threshold of the $j$-rd hidden layer nodes, $w_{1 k}, w_{2 k}, \ldots, w_{J k}, \theta_{k}$ is the connection weight between the hidden nodes and the $k$-rd output layer node and the threshold of the $k$-rd output node.

According to the improved colony algorithm is proposed in this paper, the inhibition of poor food source to become the reference source, namely, according to a certain proportion to choose better nectar source as food source. Following the selection probability $p$ of bee by the following formula:

$$
p_{i}=\frac{0.9 \times f i t_{i}}{\text { max_fit }}+0.1
$$

Among them, max $\_f i t=\max \left(f i t_{1}, f i t_{2}, \ldots, f i t_{S N}\right)$. The training steps of BP neural network based on the improved artificial bee colony is as follows:

1. To determine the structure of the neural network and target optimization problem of MSE function: the network is divided into three layers, which has $I$ input nodes, $J$ hidden nodes, $K$ output nodes.

2. The parameters of the initial artificial bee colony algorithm, including population size $S N$, which employed bee and followed bee each half; the cycle times limt of scout bees appeared; the maximum number of iterations max_gen and the preset precision target value $\varepsilon$.

3. Set the initial number of iterations gen $=0$, randomly generated $S N$ food sources, to form the initial food source of the bee colony, and each component are random numbers in the interval $(-1,1)$. Calculated for each solution's fitness function value;

4. According to a certain proportion the employed bee to choose the optimal food source as a reference for the search to generate the new $x_{i}^{\text {new }}$, and calculate the fitness value; if the new $x_{i}^{\text {new }}$ fitness value is better than the current solution is $x_{i}$, use $x_{i}^{\text {new }}$ instead of $x_{i}$, otherwise keep $x_{i}$ unchanged, and will make the retention times of $x_{i}$ add 1 ;

5. According to equation (25) calculated the probability $p_{i}$ corresponding with $x_{i}$;

6. Each food source of the followed bee's income $p_{i}$ to choose the food source, and according to a certain proportion to choose the optimal food source as a reference for the search to generate the new $x_{i}^{\text {new }}$, and calculate the fitness value; if the new $x_{i}^{\text {new }}$ fitness value is better than the current solution is $x_{i}$, use $x_{i}^{\text {new }}$ instead of $x_{i}$, otherwise keep $x_{i}$ unchanged, and will make the retention times of $x_{i}$, add 1 ;

7. Judge if whether to give up the solution, namely, if a solution is continuously through the limit times still did not improve, so will produce the scout bee, otherwise make it cross with the global optimal, generating a new solution $x_{i}$, for the corresponding update;

8. After one iteration is completed, record the best solution as so far;

9. Termination condition judgments: whether gen has already reached the maximum number of iterations max_gen or the objective function value is less than $\varepsilon$, as long as meet one of the conditions the iterative terminate and output the best solution $G$, otherwise gen $=$ gen +1 , turn to step (4).

\subsection{Convergence Precision Optimization Based on Chaos Cycle Perturbations}

In order to further improved the accuracy of the BP neural network algorithm in enterprise investment prediction, this paper then uses the chaos cycle perturbations thoughts to optimize it. 
The main means to search chaos optimization is chaos periodic disturbance in the current. Using the appropriate disturbance manner to make fully ergodic chaotic variables to obtain the optimal solution. The search process based on the chaotic is divided into two stages as follows: firstly, the generation of ergodicity track based on deterministic iterative has a disturbance on the range of the solution of the desired problem. When satisfy some termination conditions, think of the search for optimal solution of the problem has been approached, and taking this as a starting point for the next phase. Secondly, the results obtained in the first stage as the center, attached a small perturbation as the second disturbance, in order to achieve fine search in local region, until a termination condition is satisfied. Among them, the small disturbance added can be chaotic variables, can also be a random variable in other ways.

This paper uses Logistic map as the periodic disturbance factor, as shown in the following formula:

$$
x_{n+1}=\mu x_{n}\left(1-x_{n}\right)
$$

Among them, $\mu$ is the bifurcation parameter, $x_{n}$ in the interval $[0,1]$ value, that is, the map is a self map on line $[0,1]$.

After determining the network structure, the input variables and the output variables of the neural network, the training process is to find a set of weights and thresholds ,make the output values and the expected value of the neural network have a minimum error, that can be expressed as an optimization problem:

$$
\min f(\omega), \omega=\left[\omega_{1}, \omega_{2}, \ldots, \omega_{m}\right]^{T}
$$

The vector $\omega$ represent the weight value and threshold value of the neural network; $m$ is the number of elements of $\omega ; f$ is the error function corresponding to $\omega$, the following function can be used to represent:

$$
f=\frac{1}{2} \sum_{i=1}^{N}\left(d_{i}-y_{i}\right)^{2}
$$

Among them: $d_{i}$ is the expected output of the neural network; is the calculation output of the network.

This paper design the thoughts of the BP neural network based on chaos cycle perturbations is: conduct a chaotic period disturbance to get an initial weight $\omega$, and then use the BP algorithm for iterative learning, then by using chaos optimization method for the two time perturbation jump out of the local minimum to achieve the purpose of optimization.

Give a slightly different initial value $x_{0, i}(i=1,2, \ldots, m)$ on the mapping of Logistic, generate $m$ different trajectories of the chaos variables $\left\{x_{n, i}(i=1,2, \ldots, m)\right\}$, linear transform the $m$ chaotic variables into chaotic variables of solution space:

$$
\omega_{n, i}=\frac{b_{i}+a_{i}}{2}+\frac{b_{i}-a_{i}}{2} x_{n, i}
$$

A chaotic vector $\omega^{n}=\left[\omega_{n, 1}, \omega_{n, 2}, \ldots, \omega_{n, m}\right]^{T}$ in the original problem solution space is obtained.
1. If $j=0$, the target value $f^{*}=\xi$, the $x_{n}$ of the formula (26) were given $m$ small differences initial value $x_{1, i}(i=1,2, \ldots, m)$, generate chaotic vector $\omega^{j}$ according to the formula (29), in which a larger positive $\xi$ is given.

2. A chaotic periodic perturbation is used for solutions vector optimization. For the weight vector of $\omega^{j}$, calculate the value of the objective function $f\left(\omega^{j}\right)$, if $f\left(\omega^{j}\right)<f^{*}$, make the $f^{*}=f\left(\omega^{j}\right)$, and note the optimal solution by $\omega^{*}=\omega^{j}$; otherwise make $j=j+1$, get $x_{j, i}$ according to the formula (26), then generate new vector $\omega^{j}$ by substituting into formula (29) for a carrier.

3. Set the number of iterations $T$ and computational accuracy, repeat Step (2) and reach iterations set in advance or meet the calculation precision $\varepsilon$ was given, seeking solutions $\omega^{*}$ and target value $f^{*}$. If it meets the requirements, that is $f^{*} \leq \varepsilon$, and the algorithm terminates; otherwise turn to Step (4).

4. Take the $\omega^{*}$ into the BP algorithm as the initial value to train the weights and thresholds. If the learning reaches the maximum algebraic $H$ set in advance or after learning $N$ times the target value remains unchanged, then the network weights and threshold vector is denoted as $\omega$, and the corresponding target value is denoted as $f_{2}^{*}$. If $f_{2}^{*}$ meets the requirements of precision, the algorithm terminates; otherwise turn to Step (5).

5. Set $k=0$, initialize chaos vector $X_{0}$ in interval $[0,1]$ randomly; generate weights vector $\omega^{\prime}$ according to $\omega^{\prime}=\omega+\beta X_{k}$, in which $\beta$ is a given constant and can be adjusted in the calculation process; then calculate the value of the objective function $f\left(\omega^{\prime}\right)$ if $f\left(\omega^{\prime}\right)<f(\omega)$, then update the optimal value; otherwise order $k=k+1$, return type (26) and iterative generate $X_{k}$, generate the new weights vector $\omega^{\prime}$ by taking it into $\omega^{\prime}=\omega+b X_{\kappa}$; repeat the above operation to reach the maximum algebraic $H$ set in advance or meet the precision $\varepsilon$, obtaining the optimal solution $\omega^{*}$ and its corresponding target value $f_{3}^{*}$. If $f<f_{3}^{*}<f_{2}^{*}$, return to (4) to do another BP optimization; otherwise the algorithm terminates, and outputs results.

\section{The Simulation of the Algorithm Performance}

In order to verify the validity of the improved algorithm proposed in this paper, simulation experiment is carried out. First, we simulate the improved artificial bee colony algorithm, and then choose Iris data set, Wine data set and Glass data set for experiment.

The experimental parameter settings: select the number of colony food source $S N=20$; influence of parameters of scout bees appear limit $=50$; the maximum number of iterations 500; training accuracy limit $=50$.

The experimental results of artificial bee colony algorithm and the improved artificial bee colony algorithm are shown as follows. 


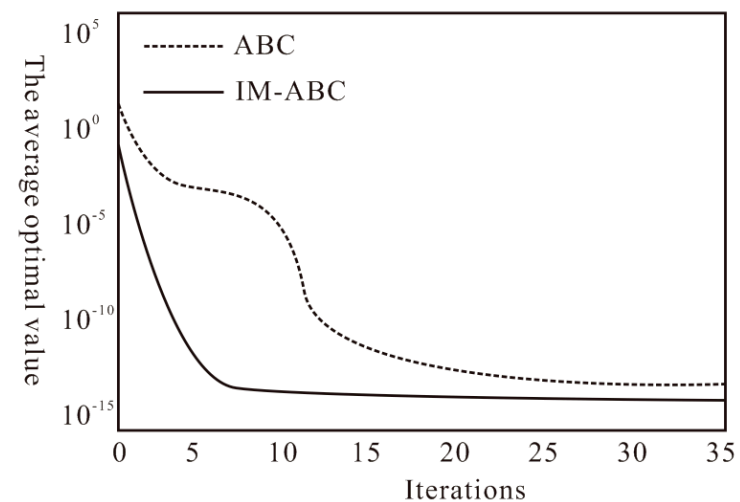

Fig. 2. Artificial bee colony algorithm for the simulation results.

From the simulation results, we can see clearly that the searching ability of the improved artificial bee colony algorithm is better than the standard artificial bee colony algorithm. Then have the simulation experiment of BP neural network based on double optimization algorithm, and the results are as follows:

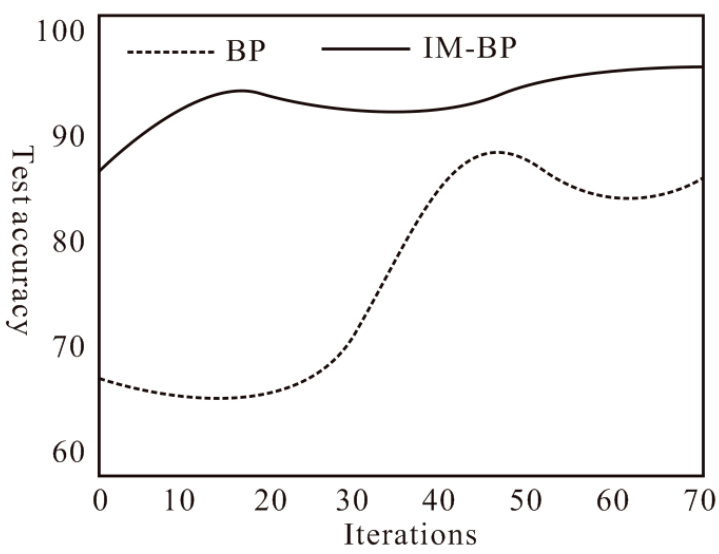

Fig. 3. Iris data set for the simulation results.

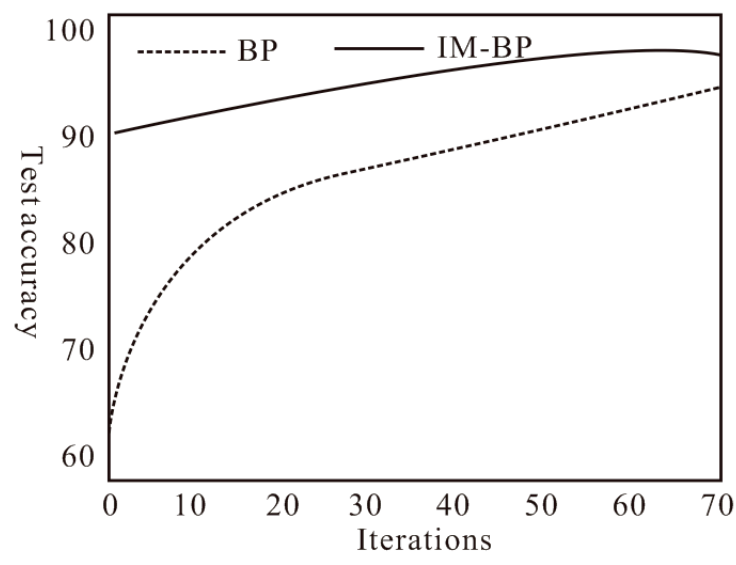

Fig. 4. Wine data set for the simulation results.

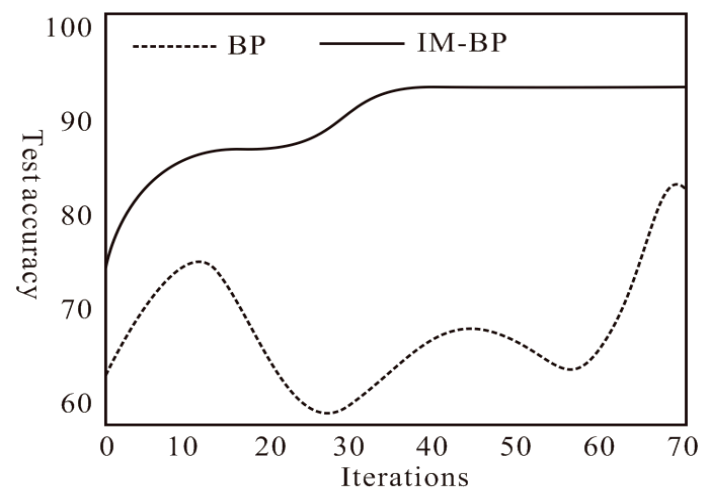

Fig. 5. Glass data set for the simulation results.

Obviously, the improved BP neural network has been greatly improved. Finally, construct the enterprise investment prediction model by adopting BP neural network based on the improved double optimization algorithm, then get the forecast error, and the results are as follows:

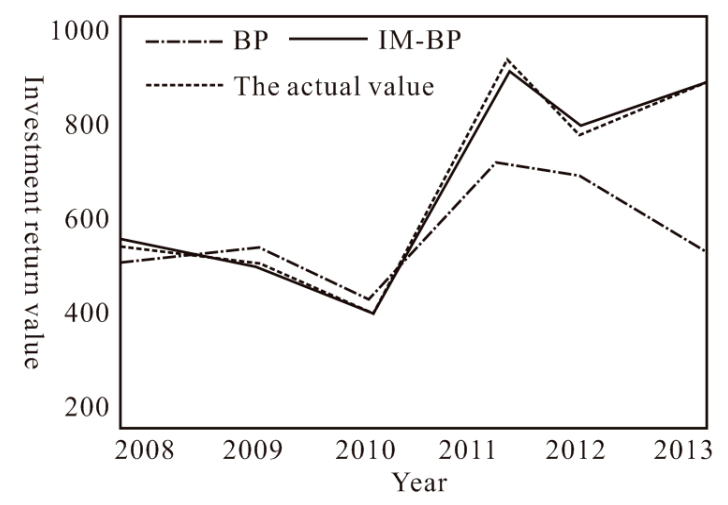

Fig. 6. Business investment forecast results.

As can be seen from simulation results, for enterprise investment, compared with the standard BP neural network algorithm, the proposed BP neural network algorithm based on the improved artificial bee colony optimization and chaos cycle perturbations has a higher forecast accuracy and a better convergence performance.

\section{Conclusion}

With the booming of the economy and the state easing of the investment and financial policy, the enterprises of our country have greatly increased the investment behavior in recent years. This paper put forward the BP neural network algorithm based on the improved artificial bee colony and chaotic periodic perturbation optimization according to the prediction model of enterprise investment's demand. We can seen from the simulation results, the algorithm for the enterprise investment forecast model has the preferable forecast precision and convergence performance. 


\section{References}

1. L. Hongchang, New decision system and investment macro control, Economic Survey, vol. 4, pp.5-11, (2014).

2. J Zhong, The fluctuation of investment and investment control from the perspective of comparative view, Shanghai Economic Research Form, vol. 2, pp. 9-14, (2014).

3. H. Feng, The research of non efficiency of investment control of state owned enterprises, Communication of Finance and Accounting, vol. 4, pp. 26-28 (2014).

4. X. Huang, Investment fluctuation and economic growthRethinking of the investment control mechanism, Economic Theory and Business Management, vol. 4, pp. 36-42 (2013).

5. Y. MA, Practice and countermeasure of strengthening financial management of construction-agent system in governmentinvested projects, Finance and Accounting, vol. 4, pp. 36-42 (2013).

6. H. WU, Research on railway freight traffic prediction based on maximum Lyapunov exponent, Journal of the China Railway Society, vol. 36(4), pp. 7-13 (2014).

7. J. Zhang, Crude oil price chaotic forecasting method based on hybrid model, Operations Research and Management Science, vol. 22(5), pp. 166-172 (2013).

8. Y. Luan, The date of bridge deformation: the wavelet denoising and chaotic forecasting, Geodesy and Geodynamics, vol. 33(5), pp. 133-135 (2013)

9. B. Liu, Chaotic prediction for network traffic based on dynamic weighted least squares support vector machine, TV Engineering, vol. 37(7), pp. 87-90 (2013).
10. W. Zhang, The chaotic prediction of natural circulation flow instability based on the Lyapunov index under the rolling condition, Journal of Physics, vol. 6, pp. 53-60 (2013).

11. X. Feng, The chaotic prediction of network traffic of RBF neural network based on wavelet packet, Computer Engineering and Design, vol. 33(5), pp. 1681-1686 (2013).

12. Y. Fan, The study on the performance of chaotic prediction of frequency hopping code sequence, Computer Applications and Software, vol. 28(8), pp. 83-86 (2013).

13. S. Li, Chaotic prediction for short-term traffic low of optimized BP neural network based on genetic algorithm, Control and Decision, vol. 26(10), pp. 1581-1585 (2013)

14. Y. Yu, A class of chaotic prediction of non smooth periodic perturbations in the Duffing system, Mathematics in Practice and Theory, vol. 41(9), pp. 239-244 (2013).

15. L. Liu, Comparative study on chaotic prediction model in shortterm traffic flow, Mathematics in Practice and Theory, vol. 41(17), pp. 106-114 (2013).

16. C. Sun, Chaotic prediction of deepwater motor system based on wavelet neural network, Journal of Shenyang University of Technology, vol. 31(1), pp. 7-10 (2013).

17. B. Wang, The detection of outlier time sequence based on chaotic prediction, Journal of Wuhan University: Engineering and Technology, vol. 43(2), pp. 265-268 (2013). 